\title{
Early oral feeding reduced recovery time after surgery for gynaecological malignancies
}

\author{
Cutillo G, Maneschi F, Franchi M, et al. Early feeding compared with nasogastric decompression after major oncologic gynecologic \\ surgery: a randomized study. Obstet Gynecol 1999 Jan;93:41-5.
}

\section{Question}

Is early oral feeding after major gynaecological oncological surgery as effective and safe as nasogastric decompression and feeding at first passage of flatus?

\section{Design}

Randomised (concealed), unblinded, controlled trial.

\section{Setting}

3 university affiliated hospitals in Italy.

\section{Patients}

122 patients who had elective laparotomy for gynaecological malignancies. Exclusion criteria were previous pelvic or abdominal radiotherapy; intestinal obstruction before surgery; gastrointestinal, breast, pancreatic, or biliary duct neoplasia diagnosed during surgery; concomitant intestinal resection; and duration of surgery $<60$ minutes.

\section{Intervention}

Patients were stratified by duration of surgery and type of tumour. 61 patients were allocated to receive early oral feeding of clear fluids on the morning of the first day after surgery and a semi-liquid fibreless diet within the next 24 hours. The diet was accelerated as tolerated to a regular diet. 61 patients were allocated to have nasogastric decompression and removal of the nasogastric tube after first passage of flatus or when gastric drainage was $<50 \mathrm{ml} /$ day. These patients received clear liquids on the day of first passage of flatus, were advanced to a semi-liquid diet within the next 24 hours, and were accelerated as tolerated to a regular diet. All patients received antibiotic prophylaxis and mechanical bowel preparation before surgery, balanced standard anaesthesia and orogastric decompression during surgery, and standard analgesia and infusional treatment after surgery. All were mobilised on the first day after surgery.

\section{Main outcome measures}

Time to passage of flatus, stool, and tolerance of regular diet; duration of stay after surgery; nausea; vomiting; and complications after surgery (fever, wound infection or dehiscence, pneumonia, ascites, deep venous thrombosis, ureteral fistula, bowel adhesion, and symptomatic lymphocyst).

\section{Main results}

Patients who received early oral feeding had a shorter time to first passage of flatus (median $2 v 3 \mathrm{~d}, \mathrm{p}<0.01$ ), first passage of stool (median $3 v 4 \mathrm{~d}, \mathrm{p}<0.01$ ), tolerance of regular diet (median $3 v 5 \mathrm{~d}, \mathrm{p}<0.01$ ), and had a shorter hospital stay after surgery (median $5 \cup 6 \mathrm{~d}, \mathrm{p}<0.05$ ) than patients who had nasogastric decompression and feeding after first passage of flatus. Groups did not differ for nausea, vomiting, or complications after surgery.

\section{Conclusions}

Early oral feeding after major gynaecological oncological surgery reduced time to first flatus, time to first stool, time to tolerance of regular diet, and duration of hospital stay after surgery compared with patients who received nasogastric decompression and oral feeding after first passage of flatus. No differences were found for nausea, vomiting, or complications.

Source of funding: no external funding.

For correspondence: Dr P Benedetti-Panici, Department of Gynaecology, University Campus BioMedico, Via Longoni 69, 00155 Rome, Italy.

\section{Commentary}

Although research to date has been limited, increased attention has recently been given to the dietary management of patients after surgery. The study by Cutillo et al is one of the first to evaluate the effect of traditional dietary management practices after surgery on patient outcomes. The authors' findings support only some of the conclusions of a previous meta-analysis of routine nasogastric decompression after laparotomy. ${ }^{1}$ The study did support the finding that $>95 \%$ of patients who had elective laparotomy could be successfully managed without a nasogastric tube after surgery.

Stratification of patients by duration of surgery and type of tumour is a strength of this study. The study participants were all women from 3 hospitals in Italy, so findings may differ from other populations. The use of clear liquids as the first meal after surgery does not reflect the findings of Jeffery et al who compared the use of clear liquids with a regular diet as the first meal after abdominal surgery and found no differences in the incidence of post-operative complications. ${ }^{2}$ The argument supporting early feeding could have been strengthened if this finding had been incorporated into the design of the study by Cutillo $e t$ al.

The results are relevant to medicalsurgical and surgical oncology nurses, nurse practitioners, and clinical nurse specialists working with surgical patients. The findings increase our understanding of the appropriate dietary management of patients after abdominal surgery. They bring into question traditional post-operative management practices. Early feeding on the first day after surgery is associated with improved patient outcomes and reduced hospital stay. Routine nasogastric tube insertion for decompression after major oncological gynaecological surgery is not warranted. Tube placement should be ordered on an individual basis only for complications such as development of an ileus. Nurses can use this knowledge to advocate more successfully for changes in post-operative practices that are evidence based, improve patient outcomes, increase patient comfort, and reduce the length of hospital stay.

Hilaire J Thompson, RN, MS, CS Nurse Practitioner University of Pennsyluania Philadelphia, Pennsylvania, USA

1 Cheatham ML, Chapman WC, Key SP, et al. A meta-analysis of selective versus routine nasogastric decompression after elective Sogastric decompression after election
laparotomy. Ann Surg 1995;221:469-78.

2 Jeffery KM, Harkins B, Cresci GA, et al. The Jeffery KM, Harkins B, Cresci GA, et al. The
clear liquid diet is no longer a necessity in the routine postoperative management of surgical patients. Am Surg 1996;62:167-70. 